\title{
Warning signals confer advantage to prey in competition with predators: bumblebees steal nests from insectivorous birds
}

\author{
Piotr G. Jablonski • Hyun Jun Cho • Soo Rim Song • \\ Chang Ku Kang • Sang-im Lee
}

Received: 26 October 2012 /Revised: 22 April 2013 / Accepted: 23 April 2013 /Published online: 24 May 2013

(C) The Author(s) 2013. This article is published with open access at Springerlink.com

\begin{abstract}
Aposematic (warning) signals of prey help predators to recognize the defended distasteful or poisonous prey that should be avoided. The evolution of aposematism in the context of predation has been in the center of modern ecology for a long time. But, the possible roles of aposematic signals in other ecological contexts have been largely ignored. Here we address the role of aposematic signals in competition between prey and predators. Bumblebees use visual and auditory aposematic signals to warn predators about their defenses. For 2 years, we observed competition for nestboxes between chemically defended insects, Bombus ardens (and possibly also Bombus ignitus), and cavity nesting birds (Parus minor and Poecile varius). Bumblebees settled in 16 and $9 \%$ of nestboxes (in 2010 and 2011 breeding seasons, respectively) that contained bird nests at the advanced stage of nest building or at the stage of egg laying. Presence of bumblebees prevented the birds from continuing the breeding activities in the nestboxes, while insects took over the birds' nests (a form of kleptoparasitism). Playback experiments showed that
\end{abstract}

Communicated by D. Rubenstein

Electronic supplementary material The online version of this article (doi:10.1007/s00265-013-1553-2) contains supplementary material, which is available to authorized users.

P. G. Jablonski $\cdot$ H. J. Cho $\cdot$ S. R. Song $\cdot$ C. K. Kang $\cdot$ S.-i. Lee Laboratory of Behavioral Ecology and Evolution, School of Biological Sciences, College of Natural Sciences, Seoul National University, Seoul 151-742, South Korea

P. G. Jablonski $(\bowtie)$

Centre for Ecological Research [formerly Institute of Ecology], Polish Academy of Sciences, Dziekanow Lesny, Poland e-mail: piotrjab@behecolpiotrsangim.org

S.-i. Lee

Institute of Advanced Machinery and Design, Seoul National University, Seoul 151-742, South Korea the warning buzz by bumblebees contributed to the success in ousting the birds from their nests. This demonstrates that aposematic signals may be beneficial also in the context of resource competition.

Keywords Auditory warning signals $\cdot$ Resource competition $\cdot$ Paridae $\cdot$ Bombus $\cdot$ Kleptoparasitism . Nestbox $\cdot$ Distant taxa $\cdot$ Parus minor $\cdot$ Poecile varius

\section{Introduction}

The evolution and ecology of conspicuous warning visual (Cott 1940; Svadova et al. 2009; Stevens and Ruxton 2011), auditory (Hristov and Conner 2005; Bura et al. 2009), or multimodal (Ratcliffe and Nydam 2008) signals in prey to deter predatory attacks through signaling the presence of defenses (chemical, mechanical, etc) is in the center of behavioral ecology (Ruxton et al. 2004; Mappes et al. 2005). Warning signals help the predators to quickly learn and remember how to recognize the defended and distasteful/poisonous prey that should be avoided. Olfactory or acoustic signals promote learning by the predator to visually avoid the aposematic prey (Rowe and Guilford 1996; Rowe and Guilford 1999; Rowe 2002; Kelly and Marples 2004; Hauglund et al. 2006). Sometimes multiple modalities, for example olfactory and visual, or acoustic and visual modalities, are used for aposematic signaling to predators because multiple modalities would enhance the effectiveness of aposematism. For example, bumblebees are not only aposematically colored but they also use warning acoustic signals when disturbed (Goulson 2010).

While the mechanisms of evolution of aposematism in the context of predation have been in the center of modern evolutionary ecology for years, the possible role of aposematic 
signals in other ecological contexts has been largely ignored. Many aposematic signals are conspicuously presented not only in response to actual predatory attack but either continuously or in response to any stimulus that may indicate danger and a possibility of predator presence. Therefore, it is expected that they will be detected by all organisms nearby the aposematically displaying individuals and in the contexts other than predation. Hence, it is likely that, in addition to protecting the prey in the context of predation, the warning signals affect other ecological interactions between signaling individuals and other organisms in the same ecosystem. Indeed, aposematic signals have been shown to affect interactions in the context of mating (Summers et al. 1999; Melo et al. 2009; Crothers et al. 2011), or oviposition (Papaj and Newsom 2005). However, we are unaware of any study on how aposematic (warning/defensive) signals that deter predatory attacks may also affect inter-specific ecological competitive interactions between the predator and prey.

An aposematic (chemically or otherwise defended) prey is often taxonomically distant from the predators that drive the evolution of aposematism (insect prey and avian predators: Harvey et al. 1982; Wiklund and Järvi 1982; Alatalo and Mappes 1996; insect prey and mammalian predators: Hristov and Conner 2005; and amphibian prey and mammalian predators: Saporito et al. 2007). Hence, if competition between the prey and predator is present in those systems, it belongs to the category of "competition between distant taxa" (Diamond 1987), which is expected to have highly asymmetric outcome (Diamond 1987; Barnes 2003). However, only a dozen or so reports focused on the competition between distant taxa (Carpenter 1979; Brown et al. 1979, 1981; Brown and Davidson 1979; May et al. 1979; Eriksson 1979; Woodin and Jackson 1979; Hill et al. 1986; Hurlbert et al. 1986; Schluter 1986; Morin et al. 1988; Englund et al. 1992), representing a tiny fraction of papers on competition (MacArthur 1972; Gilpin and Case 1976; Pimm 1978; Diamond and Case 1986; Schoener 1983; Sih et al. 1985; Goldberg and Barton 1992; Sommer and Worm 2002).

Richness of taxonomic composition of many ecological communities may create situations in which competition between distant taxa is observed. For example, in a typical temperate forest, natural tree cavities are used by an assemblage of cavity nesting birds, by bats, and by some insects including chemically defended Hymenoptera (Carlson et al. 1998). Therefore, we may expect direct competition between birds and hymenopteran insects, such like bumblebees, when tree cavities are in short supply. Here we investigated the competition between bumblebees and birds for cavities. Because bumblebees can benefit from, or may require, plant materials already present in the cavities (Fye and Medler 1954; Goulson 2010; Lye et al. 2011), we may expect that they shall try to steal freshly built nests from the birds.
Bumblebees are well defended (sting) and they signal this to their predators with warning coloration (Mostler 1935; Brower et al. 1960) and with warning auditory signals when disturbed (Mostler 1935; Kirchner and Roschard 1999; Goulson 2010). Bumblebees also use abandoned or active nests of mammals and ground or tree cavities with abandoned nests of birds (Goulson 2010; see a review of published reports in Rasmont et al. 2008) including nestboxes (Osborne et al. 2008). Therefore, one can expect that bumblebees may benefit from taking over a cavity with a freshly built nest if only they are able to oust the birds that are using the cavity. Indeed, reports of successful oustings of breeding birds by bumblebees in mixed forests of southeastern Europe (Rasmont et al. 2008), northern Europe (Orell and Ojanen 1983), and Japan (Mikami and Yamaguchi 2011) suggest that this competition may be present in temperate forests. Tits are common cavity nesters and they are known to predate on bumblebees (Haeseler 1975; Forster-Johnson 2002; Goulson 2010; Brennand 2011). Hence it appears that competition between bumblebees and birds can be categorized as competition between the prey and their predators. The behavioral mechanisms of this competition have not been studied.

Our aim was to study the mechanism and the outcome of interactions between bumblebees and cavity-nesting birds (Parus minor and Poecile varius) in nestboxes. We asked whether bumblebees attempt to settle in those boxes to which the birds brought fresh nest materials and whether their warning signals may provide an advantage in taking over the nests from birds.

\section{Methods}

\section{Study species}

The study was conducted in the slopes of the Gwanak Mountain that surround Seoul National University campus in Seoul, South Korea. Many bumblebees, including the common Korean bumblebees, Bombus ardens and Bombus ignitus, require cavities for nesting (Sakagami and Katayama 1977), and accept artificial nestboxes made for bumblebees (Yoon et al. 2002). The bumblebee nestboxes resemble bird boxes but they are smaller, have smaller entrance holes, and contain plant material for insulation (Fye and Medler 1954; Goulson 2010; Lye et al. 2011). The queens emerge and search for suitable breeding cavities around the same time when cavity nesting Oriental tits (P. minor) and Varied tits $(P$. varius $)$ approach their peak of nest building and egg laying activity (Choi et al. 2007; Hannan et al. 1997; Lee et al. 2011; Rhim et al. 2011). By providing avian nestboxes in a forest where natural cavities are rare, we observed the competition between tits and bumblebees for the nestboxes. 
Nestbox monitoring schedule

Sixty and 122 nestboxes were monitored in the mixed forests of the Gwanak Mountain (N 37 27.46 E 126 56.81; altitude between 102 and $248 \mathrm{~m}$ ) in 2010 and 2011, respectively. The size of the nestboxes was $11 \times 15 \times 25 \mathrm{~cm}$, and the entrance hole had a diameter of $3.5 \mathrm{~cm}$. They were hung $2.50-3.50 \mathrm{~m}$ above the ground on the tree trunks at least several months before the breeding season. This was a part of ecological research on tits, and nestbox checking schedule differed between the 2 years. In 2010, nestboxes were checked once in 1-2 weeks in April and May. In 2011, they were checked twice a week from early April till the end of June. The breeding status of birds in each nestbox was classified as one of the three categories: Empty—box empty or with only a small amount of mosses, the typical material used by tits (less than $1 \mathrm{~cm}$ layer, usually much less), Nest Present-advanced nest-building stage (more than $1 \mathrm{~cm}$ layer of mosses; usually almost a full nest present) or egg laying, and Bird Present - eggs or nestlings present. Categories Empty and Nest Present differed clearly in the absence/presence of plant materials required for bumblebees to set up their nests in the nestboxes, but they did not differ in terms of the long-term presence of a bird inside the box (because tits visit the nestbox with plant materials and stay for a short time during nest building, a bird is absent from those nestboxes for most of the day time). Categories Nest Present and Bird Present differed mostly in the presence/absence of a bird (incubating eggs or hatchlings), which may affect bumblebee's attempts and success in invading the nestbox.

\section{Statistical analysis of nestbox occupancy}

We treated each year separately because of different monitoring schedule (see above). In 2011, we were able to categorize the stage of nesting at the date when the bumblebee was first recorded due to frequent nestbox check. All recorded bumblebee invasions occurred between 20.04.2011 and 23.04.2011. This corresponded to about 12 weeks after the first sightings of bumblebee females actively searching for nest sites in our study area. In 2010, we did not check the nestboxes frequently but one of the visits was conducted on the 22nd of April 2010, a date similar to the dates of bumblebee invasions into nestboxes in 2011. Therefore, the nestbox category on the 22.04.2010 was used in the analysis of 2010 data, assuming that the status on this date represented the status at the moment of bumblebee intrusion (unknown to us for 2010). Two weeks after this date, during a consecutive nestbox check, no new invasions were recorded.

We used these data to compare the distribution of nestbox status categories among all nestboxes with the distribution among nestboxes where bumblebees were recorded. The presence of a bumblebee in a nestbox at a given stage (Empty, Nest Present, or Bird Present) indicates that the bumblebee invaded the nestbox at this or the earlier stage(s). To test the idea that bumblebees preferentially attempt to settle in those boxes in which nest material is already present, we compared the frequency of nestboxes with bumblebees at the stage of Empty with the nestboxes at the stage of Nest Present, i.e., when the bird's presence in the box was minimal but the substantial amount of the nest material was already present.

To evaluate the idea that bumblebees attempt to settle less often in those nestboxes in which the bird is already incubating or brooding than in those at an earlier stage, before the birds are almost constantly present in the nestbox and may defend it, we compared the frequency of nestboxes with bumblebees between nestboxes at the stage of Nest Present with the nestboxes at the stage of Bird Present.

We can be certain that the bumblebee individuals were different each year which assures the independence of our data. However, the tits were not individually marked, and we cannot exclude the possibility that some pairs of tits were recorded twice in two study years. Therefore there is some possibility of lack of independence for a (probably) small portion of our data points that might have been collected from nests of the same birds. Considering this, for each of the two hypotheses, we analyzed the data separately for each year, viewing them as two, fairly (albeit not entirely) independent, tests of the same hypothesis. Because the focal animals, whose behavior is characterized, are the bumblebees and not the tits, we considered that this partial violation of independence may not cause serious problem for our main conclusions. We used Fisher's exact test in Statistica (StatSoft-Tulsa, USA). All $P$ values are two-tailed.

\section{Playback experiments in nestboxes}

To test the idea that the warning defensive buzzing by bumblebees contributes to nest abandonment by the birds, in 2012 we conducted field experiments in which we recorded behavioral responses of incubating birds to the bumblebee buzz. However, to minimize the negative effect of research procedures on the breeding success of birds, we decided to conduct experiments in nests that were at the late incubation stage, when birds are less sensitive to disturbance. Typical daily incubation pattern of the Great tit (Parus major), a species very closely related to the Oriental tit (Päckert et al. 2005), involves long bouts of incubation, each lasting for an average 30 min (Bryan and Bryant 1999), and each followed by a short bout (on average $8 \mathrm{~min}$; Bryan and Bryant 1999) of foraging or other activities performed by a female after she leaves the nestbox. Hence, a female who just left a nest at the incubation stage is expected to 
come back within a relatively short time (about $20 \mathrm{~min}$ in our study site), to enter the box and to stay there for a relatively long time (half an hour or more in our study site, if not disturbed). We used this situation to study the effect of a sudden auditory disturbance, combined with the presence of a bumblebee model within the nest material, on the reaction of a female who just entered the nest and started the incubation session. We predicted that any sudden disturbance inside the nest may lead to the disruption of the normal incubation behavior, and that the disturbance consisting of the bumblebee buzz will affect the normal incubation behavior especially strongly.

In the 2012 breeding season, we conducted playback experiments in 11 nestboxes at the stage of late incubation/early feeding (i.e., 1-2 days old nestlings that were brooded by the bird). Each incubating female was tested in two conditions, bumblebee buzz (Buzz treatment) and bird song (Bird treatment, which serves here as a control) separated by 1-2 days. In six nests, the Buzz treatment was the first, while in the remaining five nests the Bird treatment was the first condition. In both treatments, the playback was delivered through a modified speaker (Fig. 1a). In the middle of the speaker, we glued a $1-\mathrm{cm}-$ long toothpick with a dead bumblebee onto its end. This set (speaker with a bumblebee) was hidden in the nest material, such that the bumblebee model was partially visible but the speaker was not visible to the bird (Fig. 1b). We monitored the behavior of the incubating bird using a small video camera located at the ceiling of the box and with a miniature microphone attached to the camera. For the Buzz treatment, we played the buzz of bumblebees recorded in one of the nestboxes occupied by the bumblebees in 2012. For a Bird treatment, we used songs of the birds most commonly heard in the study area: the cuckooing sound of the Little Cuckoo (Cuculus poliocephalus) in May and the vocalizations of Brown-eared bulbul (Hypsipetes amaurotis) in June. The bumblebee and bird recordings (Tsuruhiko and Michio 1996) were edited to last for $3 \mathrm{~s}$ and to play at approximately similar amplitude (according to human perception). For each playback experiment a different recording was used (different bumblebee recording or a different section of the commercially available recordings; Tsuruhiko and Michio 1996). For the graphical representation, the observations were scored in the following way: $1=$ some reaction, but not flying out; for example, raising the body and keeping it raised, going to the side of the box, going to the entrance but not flying out, pecking at the bee or nearby; $2=$ female reacted by flying out. For the statistical analysis we only used the binary variable indicating whether the playback affected the female (1) or not (0), and we used one-tailed McNemar statistics with continuity correction to test the hypothesis that the Buzz treatment affected females more strongly than the Bird treatment did.
Experiments with naïve hand-raised Oriental tits

To determine if the reaction to the bumblebee buzz, observed in the nestbox playback experiments, is innate or learned, we tested reactions of 11 hand-raised Oriental tits (age 30-60 days). The experimental birds were hand raised. They were fed with crickets (Acheta domestica), mealworms (Tenebrio molitor), silkworms (Bombyx mori), and seeds (sunflower, walnuts, and peanuts) using tweezers and feeding containers. We tested their reactions in response to the vibration of the buzz being played through the toothpick glued on a speaker (the same speaker that was used in nestbox experiment; Fig. 1a) while the tit touched the toothpick to eat a small amount of butter on the tip (Experiment 1). To determine if tits avoid bumblebees using visual cues, we presented a dead bumblebee, held in the tweezers, to the 11 hand-raised Oriental tit fledglings (Experiment 2). Each presentation lasted 30-45 s.

In both experiments, we classified the response of the bird as "+" if the bird moved away and did not approach the toothpick again (Experiment 1), or if a bird clearly avoided probing/pecking at the dead bumblebee (Experiment 2). Otherwise we scored the bird's response as "-", indicating a lack of avoidance to either auditory/tactile (Experiment 1) or visual (Experiment 2) bumblebee-specific cues. Additionally, we described the manner in which the birds behaved during those tests. The same birds were used in both experiments. All birds were first tested with the toothpick and the sound stimulus, and several days later with the dead bumblebee on a toothpick. Every day the birds were fed butter on a toothpick multiple times to erase any possible association with the experimental cue (presented only once to each bird).

\section{Results}

Analysis of nestbox occupancy

Two species of tits bred in the nestboxes in our study site: the Varied tit (P. varius) and the Oriental tit (P. major). All seven nestboxes with bumblebees were at the stage Nest Present: five were at the stage of nest building and two were at the stage of egg laying (Fig. 2). Bumblebees were detected in $21 \%$ (four nestboxes) of 19 nestboxes at the stage of "Nest Present" in 2010 (Fig. 2a), and in $12 \%$ (three nestboxes) of 25 nestboxes at the stage "Nest Present" in 2011 (Fig. 2b). Each of these intrusion events occurred in different nestboxes.

In six cases, the tits appeared to abandon the nestbox. In one box, bumblebee was no longer present 3 days after its presence was detected in the nestbox, and Varied tits laid eggs and successfully raised nestlings afterwards. In both 


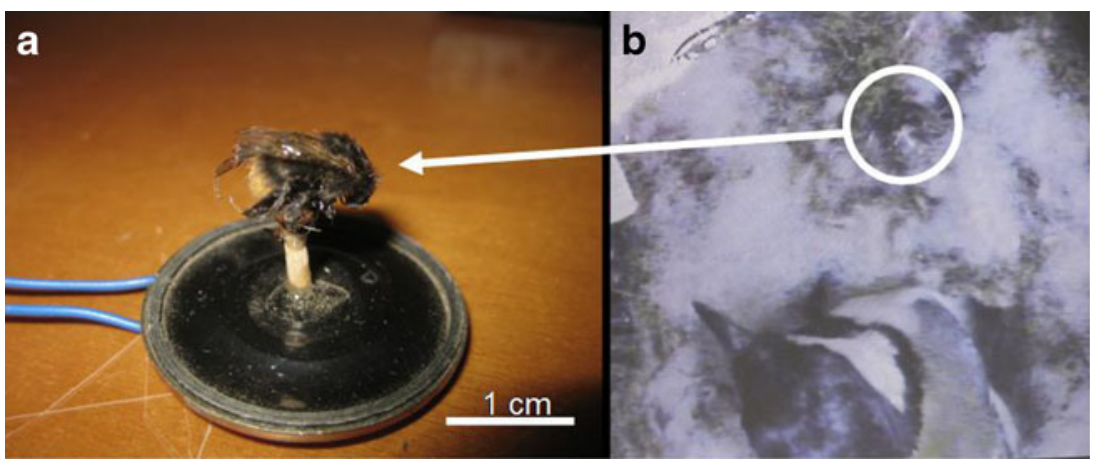

Fig. 1 Methods used in the playback experiment. a Miniature flat speaker with a dead bumblebee on a 1-cm toothpick glued onto the speaker's membrane; $\mathbf{b}$ a frame from the video recorded with a camera located above the nest in the nestbox: a female Oriental tit is at the

nests where bumblebees were found at the stage of egg laying, the eggs were abandoned by birds. Based on the features visible without capturing the bumblebees, they must have belonged to either $B$. ardens or $B$. ignitus, two similarly looking species (females look similar). B. ardens is the most common species in the study area (personal communication from S.H. Lee) and examination of several individuals by bumblebee specialists (S. H. Lee and M. R. Lee) indicated that probably all observed intrusions were by the queens of $B$. ardens (although $B$. ignitus cannot be excluded).
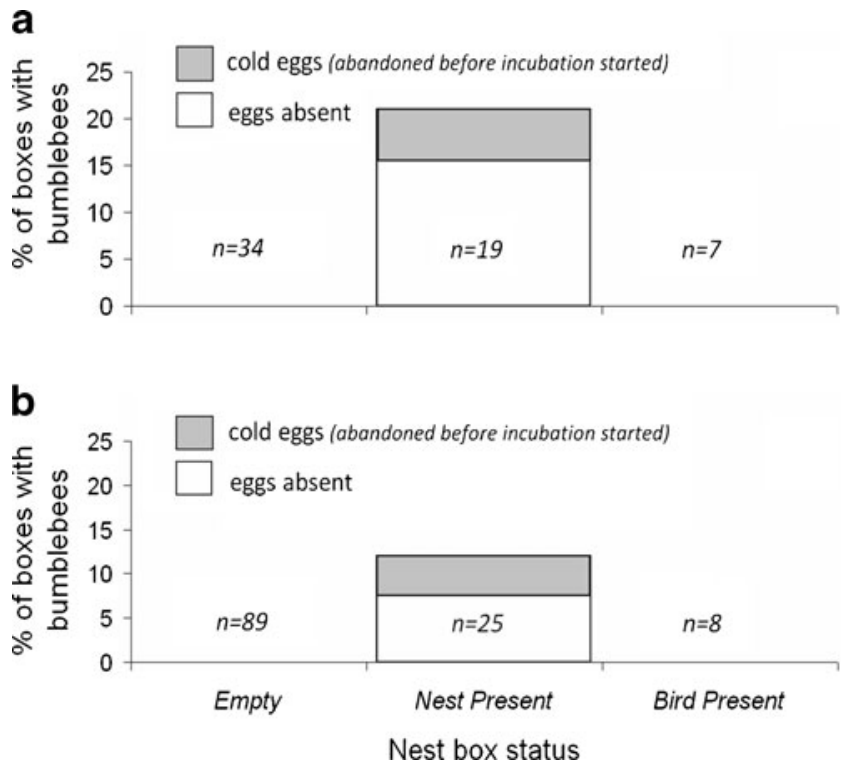

Fig. 2 Occupation by bumblebees of the nest boxes at different stages: empty box (Empty), a box with a tit nest present before incubation started (Nest Present), or a box at the stage of incubation or brooding by tits (Bird Present). a Data from 2010 breeding season; b data from 2011 breeding season. Bumblebees were detected in $21 \%$ (four nestboxes) of 19 nestboxes with bird nests before incubation occupied by birds in 2010 (Fig. 2a), and in $12 \%$ (three nestboxes) of 25 nestboxes occupied by birds in 2011 (Fig. 2b) bottom raising from the nest in response to vibrations played back through a speaker-bumblebee device hidden in the nest material (marked by a circle)

We never observed any fight or agonistic behavior, nor did we see an invading bumblebee (or any bumblebee at all) being killed by the tits. Due to short duration of our visits to the nestboxes at the early stages, and due to similarity of the egg coloration between the two tit species, we had no certainty about the tit species at the stage of nest building and egg laying for most of the boxes. However, the two species that used the nestboxes, the Varied tit and the Oriental tit, build nests that are similar in structure and the type of plant materials. Thus the nests were indistinguishable to us and could have provided similar environments for bumblebees.

In 2011, when nestboxes were monitored two to three times a week throughout the whole breeding season, we detected the first invasion by a bumblebee on 20.04.2011 and the last one on 23.04.2011. Two, out of the three, bumblebees detected in 2011 occupied nestboxes for 3 and 20 days (at least), respectively. The third nestbox was occupied by the bumblebees for at least 9 weeks, and the bumblebee female succeeded in raising a colony of workers which were observed in the nestbox on 18.05.2011 and 28.05.2011. After the monsoon season, however, the bumblebee colony disappeared from the box (no live bumblebees were present on 28.06.2011).

In accordance with the hypothesis that bumblebees attempt to settle in those boxes in which nest material is present, the presence of a well-developed bird nest was essential for occupation of a nestbox by the bumblebee (comparison between empty and nest present: Fisher's exact test $P=0.0132$ in 2010 and $P=0.0096$ in 2011; Fig. 2).

The frequencies of nestboxes with bumblebees did not differ significantly between the Nest Present and Bird Present nestboxes (Fisher's exact test; $P=0.2554$ in 2010 and $P$ $=0.4216$ in 2011). Due to a small sample of Bird Present nestboxes ( $n=7$ and $n=8$ for 2010 and 2011, respectively), this conclusion should be taken with caution. But if correct, the results do not support the hypothesis that bumblebees were more often observed in the nestboxes before 
incubation started; that is, before the birds were almost constantly present in the box (incubating or brooding) and might have attempted to defend the box when the bumblebees tried to enter.

\section{Playback experiments in nestboxes}

When the observer tried to open nestboxes that contained bumblebee females, the bumblebees buzzed in a typical defensive manner. This always caused an instinctive reflex of moving the hand away from the nest by the observer. The playback experiment tested if female birds would also be disturbed by these auditory warning signals more than by other sudden auditory signals (we used bird songs).

The sudden sound of a bird song played inside of the nestbox clearly disturbed some of the females returning to their incubation activities (Fig. 3). However, the sound of the bumblebee buzz invoked a reaction significantly more often than the bird playback did (Fig. 3; McNemar test, $\chi_{1}{ }^{2}$ $=3.200, P<0.037$ ). Most of the incubating females (nine out of 11) showed signals of distress (flying out of the nestbox or lifting its body and moving to the edge of the nest away from the source of sound; Fig. 3) in response to the bumblebee buzzing sound, and only four females responded in this way also to the sudden bird song played within the nestbox. Most females did not pay attention to the bumblebee until the buzz was played. Only one female started attacking the bumblebee by pecking it vigorously right after entering the box before any playback was conducted. It was unusually bright inside of this particular nestbox.

Experiments with naïve hand-raised Oriental tits

All ten out of 11 naïve hand-raised Oriental tits, that were sufficiently tame to frequently accept food items held by the experimenter, were not bothered by the buzzing through the toothpick. They continued eating the butter presented to

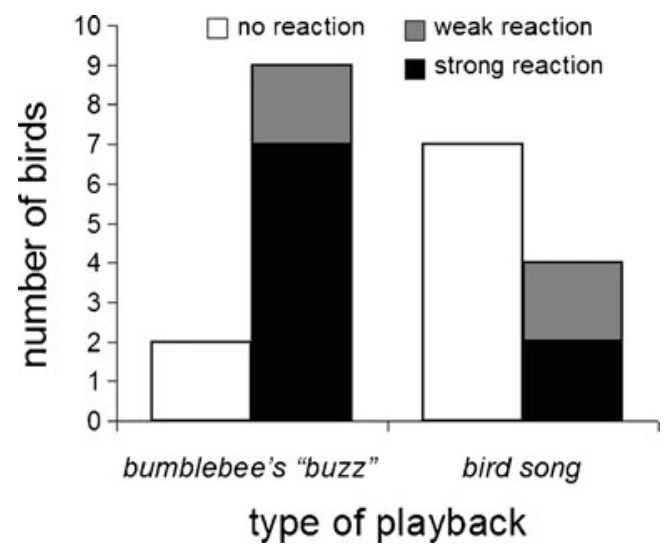

Fig. 3 Reaction of incubating females to the playback of bumblebee buzz and to the playback of a common bird song in their nests them on the toothpick connected to the membrane of the speaker when the buzz was played. The same 11 tits, while eating the butter from the tip of the toothpick, were never bothered by the bird vocalization played through the loudspeaker to which the toothpick was attached. All of the 11 hand raised Oriental tits were not scared by the presentation of the dead bumblebee in the tweezers, and proceeded to peck on its wings, thorax, abdomen, or head in a manner similar to pecking a mealworm presented in the tweezers. In summary, the two experiments showed that naïve birds were not bothered by the auditory or visual cues that contain bumblebee-specific warning signals.

\section{Discussion}

Based on the previous reports of bumblebees ousting birds from nestboxes (Orell and Ojanen 1983; Rasmont et al. 2008; Mikami and Yamaguchi 2011), we have hypothesized that bumblebee defenses and the associated aposematic acoustic signals may help them to oust birds from their nests. Our results have shown that indeed the acoustic signals (buzz) from the disturbed bumblebees may be crucial for the insects' success in this inter-specific competition for nest sites between the prey and the predator. Aposematic (warning/defensive) signals that deter predatory attacks have already been shown to affect intra-specific interactions in the context of mating (Melo et al. 2009), intra-specific competition (Crothers et al. 2011), or oviposition (Papaj and Newsom 2005). Our study adds a new context to this list: inter-specific competition between the prey and the predator.

This is the fourth scientifically documented report of bumblebees directly stealing from birds their freshly built nests in the nestboxes. The three or possibly four species of bumblebees, Bombus niveatus in Turkey (Rasmont et al. 2008), unidentified Bombus sp. in Finland (Orel and Ojanen 1983), B. ardens in Japan (Mikami and Yamaguchi 2011) and in Korea (this study), and possibly also B. ignitus in Korea (this study), all act like competitors or kleptoparasites (Brockman and Barnard 1979), who steal freshly built nests from birds. Because most invasions observed in our study and all invasions observed by Rasmont et al. (2008), Orell and Ojanen (1983)), and Mikami and Yamaguchi (2011) were associated with nest abandonment by birds, this kleptoparasitic interaction seems to lead to a clear decrease in the fitness of birds. This outcome is also consistent with asymmetrical nature of competition between distant taxa predicted by Diamond (1987) and recently confirmed by Barnes (2003) for marine taxa.

How common may this competition and its asymmetric outcome be in nature? It has been known that occasionally bumblebees use bird boxes (for example less than $5 \%$ of natural cavities in Carlson et al. 1998; about $3 \%$ of 
nestboxes in Langowska et al. 2010; 6-20\% nestboxes in Smetana and Miles 1993), but usually no information exists whether it is a result of ousting of the birds or settling in an unused box. It is possible that at least some of those cases are an outcome of direct competition between bumblebees and cavity nesting birds. On the other hand, it is likely that unsuccessful attempts of bumblebees remain unnoticed (see example in Supplemental Movie and Supplemental Text) and unaccounted for, which may lead to underestimation of the frequency of these competitive interactions and to a bias in the evaluation of their outcome.

What is the mechanism underlying the bumblebees' success? We did not observe bumblebees in the nestboxes at the stage of incubation and brooding by birds. In Rasmont et al.'s (2008) study, most bumblebee (B. niveatus) invasions of the nestboxes also occurred at the stage of nest building, when birds are present in the boxes for a short time, which may allow the bumblebee to enter the nest easily. However, the lack of statistical support for the effect of bird presence on the occupation of nestboxes by bumblebees in our data is consistent with Rasmont et al.'s observations of some bumblebees (six out of 19) invading the nestboxes even at the stage of incubation or brooding, when birds usually stay in the nestbox for a large portion of day time. Like in the previous studies (Orell and Ojanen 1983; Rasmont et al.'s 2008; Mikami and Yamaguchi 2011) the invaded nestboxes were abandoned by birds, and no indication of bird aggression towards, or consumption of, the bumblebees by tits was observed by us (no dead bumblebees found in the nest as well). Therefore, we suspect that the birds avoided the nestbox invaded by the bumblebee.

What could contribute to the success of bumblebees? Adult birds of many insectivorous bird species avoid aposematically signaling Hymenoptera (Mostler 1935). Our nestbox playback experiments showed that the warning auditory signals given by a disturbed bumblebee may play a prominent role in mediating bumblebee's success in taking over the nest from birds. In this context of dark cavities and bumblebees being hidden in the nest material, the visual aposematic signals cannot enhance the auditory aposematic signals (Rowe and Guilford 1999; Rowe 2002; Hauglund et al. 2006). Instead, the buzz alone appears to have an effect on the birds through auditory and tactile (nest material vibration) channels.

Can this result be a simple effect of the bird songs being well known and the buzz sounds being novel to the birds? If an incubating female suddenly hears a totally novel sound inside of her nestbox, it is expected that she will be alerted. However, we believe that both signals, and also sounds similar to the signals used in the experiments, are known to the wild birds from their previous experience. The song of the birds used in the experiments can be heard almost every day during a certain time of the breeding season in the study area. Similarly, the buzz of bumblebees, and sounds very similar to it, can also be heard by birds in the same habitat, especially early in the breeding season when several species of trees and bushes are in a full bloom and attract insects. Therefore, it is unlikely that the stronger reaction to the bumblebee recording was caused by novelty of this stimulus to the birds.

Naïve birds in our experiments did not seem to show any avoidance of novel auditory warning signal (nor visual signal) presented to them by the experimenter. Similar lack of avoidance of aposematically colored Hymenoptera, including bumblebees, by naïve insectivorous birds have been already shown by Mostler (1935). Therefore, we hypothesize that adult birds to whom the buzzing sound was played in the field were disturbed by the auditory warning signal because they have learned the association between the auditory signal and the risk of being stung by this Hymenopteran species. It is well known that repetitive disturbance at bird nests at the early stages of the nest cycle may lead to nest abandonment (Martin and Geupel 1993). We suggest that the bird sensitivity to disturbance at the nest and to the warning bumblebee buzz might have been exploited by several bumblebee species reported to take over nests from birds (Orell and Ojanen 1983; Rasmont et al. 2008; Mikami and Yamaguchi 2011). It is possible that the warning auditory signals not only help bumblebees in competition with their avian predators but also in competition for cavities with variety of other animals,

In summary, we have provided evidence that an aposematic signal known to help deterring predatory attacks on a potentially harmful prey may also help the prey to win ecological competition with their predators. The results suggest that similar ecological effects of aposematic signals may be present in other predator-prey systems where predator and its aposematic prey compete for the same resources.

Acknowledgments Financial support from National Research Foundation of Korea NRF-2010-0025546, NRF-2009-0082824, SNU College of Natural Sciences 3344-20110067. We thank the following friends for help in the field work and for comments: Jin-Won Lee, Larisa Lee Cruz, Byoungsoon Jang, Won Young Lee, Choongwon Jeong, Jong-Yeol Moon, Jae Hak Son, Kyung-sun Seo, Ki-san Lee, and Se-young Park. Two female Oriental tits, out of 11 used in the playback tests, came from the campus population of Oriental tits studied by the Wildlife Ecology \& Management Laboratory at the Dept. of Forest Sciences, CALS, Seoul National University, and we thank Sungjin Park, Se-young Park, Hwayeon Kang, Minsu Jeong, and Prof. Woo Shin Lee for access to those two nestboxes. We thank Prof. Seung Hwan Lee from the Seoul National University and Prof. Myeong-Ryeol Lee from the Rural Development Administration for help in identification of the bumblebee specimens.

Contribution HJC monitored nestboxes in 2011; PGJ, CGK, and SIL monitored nestboxes in 2010; PGJ and HJC conducted field playback experiment in nestboxes; SRS, PGJ, and SGL conducted the experiments on hand-raised tits; and HJC, PGJ, and SIL analyzed the data. All authors contributed to the manuscript after it was written by PGJ. 
Ethical standards The observations and experiments comply with the current laws of the South Korea, including compliance with a local permit to conduct research from the Gwanak district in Seoul

Open Access This article is distributed under the terms of the Creative Commons Attribution License which permits any use, distribution, and reproduction in any medium, provided the original author(s) and the source are credited.

\section{References}

Alatalo RV, Mappes J (1996) Tracking the evolution of warning signals. Nature 382:708-710

Barnes DKA (2003) Competition asymmetry with taxon divergence. Proc R Soc Lond B Bio 270:557-562

Brennand E (2011) Early birds catch bees not worms. BBC News. Science \& Environment. 21 April 2011, http://www.bbc.co.uk/ news/13160693

Brockman HJ, Barnard CJ (1979) Kleptoparasitism in birds. Anim Behav 27:487-514

Brower LP, Brower JVZ, Westcott PW (1960) Experimental studies of mimicry. 5. The reactions of toads (Bufo terrestris) to bumblebees (Bombus americanorum) and their robberfly mimics (Mallophora bomboides), with a discussion of aggressive mimicry. Am Nat 94:343-355

Brown JH, Davidson DW (1979) An experimental study of competition between seed eating desert rodents and ants. Am Zool 19:1129-1145

Brown JH, Davidson DW, Reichman OJ (1979) An experimental study of competition between seed-eating desert rodents and ants. Am Zool 19:1129-1143

Brown JH, Kodric-Brown A, Whitham TG, Bond HW (1981) Competition between hummingbirds and insects for the nectar of two species of shrubs. Southwest Nat 26:133-145

Bryan SM, Bryant DM (1999) Heating nest-boxes reveals an energetic constraint on incubation behaviour in Great tits, Parus major. Proc R Soc Lond B 266:157-162

Bura VL, Fleming AJ, Yack JE (2009) What's the buzz? Ultrasonic and sonic warning signals in caterpillars of the great peacock moth (Saturnia pyri). Naturwissenschaften 96:713-718

Carlson A, Sandstrom U, Olsson K (1998) Availability and use of natural tree holes by cavity nesting birds in a Swedish deciduous forest. Ardea 86:109-119

Carpenter FL (1979) Competition between hummingbirds and insects for nectar. Am Zool 19:1105-1114

Choi CY, Nam HY, Lee EJ, Chung OS, Park YS, Lee JK, Hyun JY, Lee WS (2007) Nest box preference by secondary cavity-nesting birds in forested environments. J Ecol Field Biol 30:49-56

Cott HB (1940) Adaptive coloration in animals. Methuen, London

Crothers L, Gering E, Cummings M (2011) Aposematic signal variation predicts male-male interactions in a polymorphic poison frog. Evolution 65:599-605

Diamond J (1987) Competition among different taxa. Nature 326:441

Diamond J, Case TJ (1986) Community ecology. Harper and Row, New York

Englund G, Johansson F, Olsson TI (1992) Asymmetric competition between distant taxa: poecilid fishes and water striders. Oecologia 92:498-502

Eriksson MOG (1979) Competition between freshwater fish and Goldeneyes Bucephala clangula (L.) for common prey. Oecologia 41:99-107

Forster-Johnson AP (2002) Bumblebee queens form part of the spring diet of the Great tit (Parus major L.) in southern Finland. Entomol Mon Mag 138:138
Fye RE, Medler TJR (1954) Field domiciles for bumblebees. J Econ Entomol 47:672-676

Gilpin ME, Case TJ (1976) Multiple domains of attraction in competition communities. Nature 261:40-42

Goldberg DE, Barton AM (1992) Patterns and consequences of interspecific competition in natural communities: a review of field experiments with plants. Am Nat 139:771-801

Goulson D (2010) Bumblebees: their behavior and ecology. Oxford University Press, Oxford

Haeseler V (1975) Soziale und solitäre Bienen (Apoidea) als Nahrung der Kohlmeise (Parus major L.). J Appl Entomol 78:139-150

Hannan MA, Maeda Y, Hoshikawa K (1997) Colony development of two species of Japanese bumblebees Bombus ignitus and Bombus hypocrita reared under artificial condition (Hymenoptera, Apidae). Jpn J Entomol 65:343-345

Harvey PH, Bull JJ, Pemberton M, Paxton RJ (1982) The evolution of aposematic coloration in distasteful prey: a family model. Am Nat 119:710-719

Hauglund K, Hagen SB, Lampe HM (2006) Responses of domestic chicks (Gallus gallus domesticus) to multimodal aposematic signals. Behav Ecol 17:392-398

Hill D, Wrigh R, Street M (1986) Survival of Mallard ducklings Anas platyrhynchos and competition with fish for invertebrates on a flooded gravel quarry in England. Ibis 129:159-167

Hristov NI, Conner WE (2005) Sound strategy: acoustic aposematism in the bat-tiger moth arms race. Naturwissenschaften 92:164-9

Hurlbert SH, Loayza W, Moreno T (1986) Fish-flamingo plankton interactions in the Peruvian Andes. Limnol Oceanogr 31:457-468

Kelly DJ, Marples NM (2004) The effects of novel odour and colour cues on food acceptance by the zebra finch, Taenipygia guttata. Anim Behav 68:1049-1054

Kirchner WH, Roschard J (1999) Hissing in bumblebees: an interspecific defence signal. Insect Soc 46:239-243

Langowska A, Ekner A, Skorka P, Tobolka M, Tryjanowski P (2010) Nest-site tenacity and dispersal patterns of Vespa crabro colonies located in bird nest-boxes. Sociobiology 56:375-382

Lee JK, Chung OS, Lee WS (2011) Altitudinal variation in parental provisioning of nestling varied tits (Poecile varius). Wilson $\mathrm{J}$ Ornithol 123:283-288

Lye GC, Park KJ, Holland JM, Goulson D (2011) Assessing the efficacy of artificial domiciles for bumblebees. J Nat Conserv 19:154-160

MacArthur RH (1972) Geographical ecology: patterns in the distribution of species. Harper and Row, New York

Mappes J, Marples N, Endler JA (2005) The complex business of survival by aposematism. Trends Ecol Evol 20:598-603

Martin TE, Geupel GR (1993) Nest-monitoring plots: methods for locating nests and monitoring success. J Field Ornithol 64:507-519

May RM, Beddington JR, Clark CW, Holt SJ, Laws RM (1979) Management of multispecies fisheries. Science 205:267-277

Melo MC, Salazar C, Jiggins CD, Linares M (2009) Assortative mating preferences among hybrids offers a route to hybrid speciation. Evolution 63:1660-1665

Mikami K, Yamaguchi N (2011) Displacement of great tits Parus major and varied tits $P$. varius from their nest boxes by breeding bumble bees Bombus ardens. Strix 27:53-61

Morin PJ, Lawler SP, Johnson EA (1988) Competition between aquatic insects and vertebrates: interaction strength and higher order interactions. Ecology 69:1401-1409

Mostler G (1935) Beobachtungen zur Frage der Wespenmimikry. Z Morphol Oekol Tiere 29:381-454

Orell M, Ojanen M (1983) Breeding success and population dynamics in a northern Great tit Parus major population. Ann Zool Fenn 20:77-98

Osborne JL, Martin AP, Shortall CR, Todd AD, Goulson D, Knight ME, Hale RJ, Sandersen RA (2008) Quantifying and comparing bumblebee nest densities in gardens and countryside habitats. J Appl Ecol 45:784-792 
Päckert M, Martens J, Eck S, Nazarenko AA, Valchuk OP, Petri B, Veith M (2005) The great tit (Parus major) - a misclassified ring species. Biol J Linn Soc 86:153-174

Papaj DR, Newsom GM (2005) A within-species warning function for an aposematic signal. Proc R Soc Lond B 272:2519-2523

Pimm SL (1978) An experimental approach to the effects of predictability on community structure. Am Zool 18:797-808

Rasmont P, Ayetkin AM, Kacar MS (2008) Ousting of the common redstart (Aves: Tudidae: Phoenicurus phoenicurus) from its nests by the bumblebee Bombus niveatus vorticosus (Hymenpotera: Apidae). Ann Soc Entomol Fr 44:251-255

Ratcliffe JM, Nydam ML (2008) Multimodal warning signals for a multiple predator world. Nature 455:96-99

Rhim SJ, Son SH, Kim KJ (2011) Breeding ecology of tits (Parus spp.) using artificial nestboxes in a coniferous forest over a five-year period. For Sci Technol 7:141-144

Rowe C (2002) Sound improves visual discrimination learning in avian predators. Proc R Soc Lond B 269:1353-1357

Rowe C, Guilford T (1999) The evolution of multimodal warning displays. Evol Ecol 13:655-671

Rowe C, Guilford T (1996) Hidden colour aversions in domestic chicks triggered by pyrazine odours of insect warning displays. Nature 383:520-522

Ruxton GD, Sherrat TN, Speed MP (2004) Avoiding attack (the evolutionary ecology of crypsis, warning signals and mimicry). Oxford University Press Inc., New York

Sakagami SF, Katayama E (1977) Nests of some Japanese bumblebees (Hymenoptera, Apidae). Journal of the Faculty of science Hokkaido University Series VI Zoology 21:92-153

Saporito RA, Zuercher R, Roberts M, Gerow KG, Donnelly MA (2007) Experimental evidence for aposematism in the dendrobatid poison frog Oophaga pumilio. Copeia 2007:1006-1011
Schluter D (1986) Character displacement between distant related taxa? Finches and bees in the Galapagos. Am Nat 127:95-102

Schoener TW (1983) Field experiments on interspecific competition. Am Nat 122:240-285

Sih A, Crowley P, McPeek M, Petranka J, Strohmeier K (1985) Predation, competition, and prey communities: a review of field experiments. Annu Rev Ecol Evol S 16:269-311

Smetana V, Miles P (1993) Some notes to nesting of the bumblebees (Hymenoptera, Bombidae) in bird boxes in a territory of the Krkonose. Opera Corcontica 30:175-178

Sommer U, Worm B (2002) Competition and coexistence, vol 161, Ecological studies. Springer, Berlin

Stevens M, Ruxton GD (2011) Linking the evolution and form of warning coloration in nature. Proc R Soc Lond B 279:417426

Summers K, Symula R, Clough M, Cronin T (1999) Visual mate choice in poison frogs. Proc R Soc Lond B 266:2141-2145

Svadova K, Exnerova A, Stys P, Landova E, Valenta J, Fucikova A, Socha R (2009) Role of different colours of aposematic insects in learning, memory and generalization of naïve bird predators. Anim Behav 77:327-336

Tsuruhiko K, Michio M (1996) The songs and calls of 333 birds in Japan, volume 2: songbirds. Shogakukan Inc., Tokyo

Wiklund C, Järvi T (1982) Survival of distasteful insects after being attacked by naive birds: a reappraisal of the theory of aposematic coloration evolving through individual selection. Evolution 36:998-1002

Woodin SA, Jackson JBC (1979) Interphyletic competition among marine benthos. Am Zool 19:1029-1043

Yoon HJ, Kim SE, Kim YS (2002) Temperature and humidity favorable for colony development of the indoor-reared bumblebee, Bombus ignitus. Appl Entomol Zool 37:419-423 